\title{
Solución del problema de ruteo capacitado considerando efectos ambientales mediante una técnica híbrida
}

\author{
CVRP solution considering environmental effects using a hybrid technique. \\ Luis Fernando Galindres Guancha ${ }^{1}$, Eliana Mirledy Toro Ocampo ${ }^{2}$, Antonio Escobar Zuluaga ${ }^{1}$ \\ ${ }^{1}$ Ingeniero de Sistemas y Computación, Grupo de Investigación: Grupo de Planeamiento de \\ Sistemas Eléctricos, Universidad Tecnológica de Pereira, Pereira, Colombia \\ ${ }^{2}$ Ingeniero Industrial, Grupo de Investigación: GAOPE (Grupo de aplicaciones en optimización y \\ procesos Estocásticos) Universidad Tecnológica de Pereira, Pereira, Colombia \\ lugaleutp.edu.co \\ elianam@utp.edu.co \\ aescobareutp.edu.co
}

\begin{abstract}
Resumen-En este artículo se propone el uso de una metodología híbrida para resolver el problema de ruteo de vehículos capacitado CVRP (Capacitated Vehicle Routing Problem) considerando efectos ambientales. El problema se resuelve bajo la estrategia de rutear primero y asignar después. La etapa de ruteo se resuelve de forma exacta mediante un modelo lineal entero. La etapa de asignación se realiza aplicando un algoritmo genético modificado que considera la secuencia encontrada en la etapa anterior como punto de partida para generar la población inicial. La función objetivo a optimizar corresponde a la minimización de distancia-peso recorrido a lo largo de la ruta, como indicador de impacto ambiental. La metodología se valida con casos de prueba de la literatura especializada.
\end{abstract}

Palabras clave- Algoritmo Memético, Efectos ambientales, Metodología híbrida, Problema de minimización de combustible, Problema de ruteo capacitado, Ruteo verde.

Abstract - This article proposes the use of a hybrid methodology for solving CVRP (Capacitated Vehicle Routing Problem) considering environmental effects. The problem is solved under route-first, cluster-second. The routing step is solved exactly using an integer linear model. The assigning step is performed by applying a modified genetic algorithm that considers the sequence found in the above step as a starting point to generate the initial population. Optimize the objective function corresponds to the minimization of weight-distance travel along the route, as an indicator of environmental impact. The methodology is validated with test cases from the literature.

Key Word - Memetic Algorithm, enviromental effects, hybrid methodology, Fuel Consumption Problem, Capacitated Vehicle Routing Problem, Green Vehicle Routing Problem.

\section{INTRODUCCIÓN}

El cambio climático es uno de los grandes desafíos de nuestro tiempo. Las autoridades gubernamentales y en general los consumidores de todo tipo de mercados están interesados en calcular y reducir su huella de carbono en el planeta. Las organizaciones hablan insistentemente de planear sus operaciones pero desarrollando sus actividades desde la sostenibilidad tanto financiera como ambiental.

En el gerenciamiento de la cadena de suministro verde el principal objetivo es la coordinación de todas las actividades de forma que se atiendan los requerimientos de los clientes a costo mínimo. Uno de los aspectos a considerar en la sostenibilidad de la empresa corresponde al desarrollo de sus actividades causando el menor impacto posible en su entorno. El transporte se constituye en una actividad que requiere de especial atención debido a la generación de gases de efecto 
invernadero que impactan de forma directa el medio ambiente. Por tanto se debe garantizar una operación eficiente de los vehículos vinculados a la cadena de suministro a fin de que su efecto contaminante sea minimizado.

En la literatura especializada se ha definido el G-CVRP del inglés Green Capacitated Vehicle Routing Problem [1,2]. En este problema se considera una red de distribución que consta de un depósito con capacidad limitada de mercancías que debe atender un conjunto de clientes con demanda conocida y dispersos geográficamente. El objetivo es atender todos los clientes con una flota de vehículos de capacidad homogénea a un costo mínimo pero adicionalmente garantizando el mínimo consumo de combustible.

El problema G-CVRP es NP-duro, razón por la cual se propone una metodología híbrida. La técnica propuesta está enmarcada dentro de la filosofía rutear primero- asignar después, que consiste en encontrar inicialmente un tour gigante donde se consideran todos los nodos este procedimiento se realiza resolviendo un TSP (Travel Salesman Problem) mediante un algoritmo exacto. A partir de la secuencia encontrada se realiza la segmentación de las rutas utilizando un algoritmo memético.

La siguiente estructura se sigue en este artículo: La sección 2 presenta el problema de CVRP y modelos matemáticos que lo representan. En la sección 3 se presenta una breve revisión del estado del arte sobre los factores que se consideran en el consumo de combustible y como se ha involucrado este aspecto en el CVRP. En la sección 4 se presenta un ejemplo numérico comparativo de la solución del problema con y sin considerar efectos ambientales. En la sección 5 se presenta la metodología propuesta, luego se presentan los resultados y finalmente aparecen las conclusiones, recomendaciones y trabajos futuros.

\section{PROBLEMA DE RUTEO DE VEHÍCULOS CAPACITADO}

El CVRP consiste en encontrar una serie de circuitos (cada uno corresponde a una ruta) con un costo mínimo, definido como la suma de costos de los arcos pertenecientes al circuito con las siguientes características:

i) Cada ruta inicia y termina en el depósito.

ii) Cada vértice se visita una única vez.

iii) La suma de las demandas de los vértices no debe exceder la capacidad del vehículo asignado.
El CVRP puede ser descrito como un problema teórico de grafos. Sea $G=(V, A)$ un grafo completo, donde $V=\{0, \ldots, n\}$ es el conjunto de vértices y $\delta(A)$ es el conjunto de arcos. Los vértices $i=1, \ldots, n$ corresponde a los consumidores, donde el vértice 0 corresponde al depósito.

Un costo no negativo $c_{i j}$ es asociado con cada arco $(i, j)$ que pertenece a $A$ y representa el costo en el que se incurre de viajar desde el vértice $i$ hasta el vértice $j$. Generalmente el uso de ciclos cerrados $(i, i)$ no es permitido y se impone por definición para todos los vértices. Si $G$ es un grafo dirigido el costo de la matriz es asimétrico y corresponde al problema asimétrico de CVRP denominado ACVRP. En caso contrario, se tiene que $C_{i j}=C_{j i}$ para todo $(i, j)$ que pertenece a $A$, se denomina problema simétrico capacitado, SCVRP(Simmetric Capacitated Vehicle Routing Problem ). El conjunto de arcos $A$ es generalmente remplazado por un conjunto de arcos no dirigidos, $E$. El grafo $G$ debe ser fuertemente conectado y generalmente se asume completo.

En casos prácticos, la matriz de costos satisface la desigualdad triangular que se expresa en la ecuación (1).

$c_{i k}+c_{k j} \geq c_{i j} \quad \forall i, j, k \in V$

En otras palabras no es conveniente desviarse de la dirección directa entre los vértices $i$ y $j$. La presencia de la desigualdad triangular algunas veces se requiere para algoritmos del CVRP.

Cada consumidor está asociado con una demanda no negativa conocida $d_{i}$ que debe ser despachada y el depósito tiene una demanda ficticia $d_{o}=0$. Dado un conjunto de vértices $S \underline{c} V$, donde la totalidad de la demanda del conjunto se expresa como la sumatoria de las demandas de cada nodo del conjunto, según la ecuación (2).

$$
d(S)=\sum_{i \in S} d_{i}
$$

Un conjunto de $K$ idénticos vehículos, cada uno con capacidad $Q$ disponibles en el depósito. Para asegurar la factibilidad se asume que $\mathrm{d}_{\mathrm{i}} \leq Q$ para cada cliente $i$. Cada vehículo puede ejecutar al menos una ruta y se asume que $k$ no es más pequeño que el $k$ mínimo, donde el $k$ mínimo puede ser usado para resolver el problema de empaquetamiento denominado BPP del inglés Bin Packing Problem asociado con el CVRP, el que se usa para determinar el mínimo número de vehículos cada uno con capacidad $Q$, que se requieren cargar todos los $n$ ítems, cada uno con un peso no negativo $d_{i}=1, \ldots, n$.

Dado un conjunto $S \subseteq V$ donde todos los vértices tienen demanda diferente de cero, $r(S)$ es el mínimo número de 
vehículos necesario para atender a todos los consumidores en $S$. El valor de la solución óptima para el problema BPP está asociado con el conjunto de elementos de $S$, que se expresa como el límite inferior de la demanda del subconjunto de clientes dividido sobre la capacidad disponible de uno de los vehículos, como se expresa en la ecuación (3).

$$
r(S)=\lceil d(S) / Q\rceil
$$

\section{A. Modelos matemáticos para el CVRP}

Tres diferentes modelos básicos han sido propuestos para el VRP en la literatura. Los modelos del primer tipo son conocidos como VFF (Vehicle flow formulation), donde se usan variables enteras asociadas con cada arco del grafo, el cual contiene el número de veces que el arco es atravesado por un vehículo. La segunda familia de modelos se denomina $\mathrm{CFF}$ (commodity flow formulation), en este tipo de modelos además de las variables enteras asociadas a los arcos o aristas, se representa el flujo de mercancías a través de las rutas viajadas por los vehículos. Finalmente las Formulaciones que dividen el problema en subconjuntos, SPP (Set Partitioning Problem), Estos modelos tienen un número exponencial de variables binarias, cada una asociada con diferentes rutas factibles. Los modelos se denominan como SPP debido a que se deben agrupar las rutas con costo mínimo, los cuales atienden al consumidor una única vez y posiblemente satisfacen restricciones adicionales. $\mathrm{La}$ principal ventaja de este tipo de modelos es que permite cuantificar los costos de las rutas, dependiendo de la secuencia de la ruta y del tipo de vehículo [3].

En este artículo se presenta una explicación del modelo VFF, con base en este se establece la metodología planteada.

Formulaciones basadas en flujos, VFF (Vehicle flow formulations): Este tipo de modelos usa variables enteras, asociadas con cada arco o con cada arista del grafo, que cuentan el número de veces que el vehículo pasa por el arco. Este tipo de modelo es el que se usa con más frecuencia en las versiones básicas de VRP. Estos modelos se adaptan para los casos en que el costo de la solución puede ser expresado como la suma de los costos asociados a los arcos y cuando las restricciones más relevantes pueden representarse mediante relaciones entre el conjunto de arcos y los costos asociados a los arcos. De otro lado los modelos VFF son útiles en aplicaciones prácticas, por ejemplo cuando se debe asignar un tipo de vehículo a la ruta [3].
El modelo matemático que representa esta formulación considera la variable $x_{i j}$ que toma el valor de 1 si el arco $(i, j)$ $\epsilon$ A hace parte de la solución óptima y toma el valor de cero en caso contrario, $c_{i j}$ representa el costo de desplazarse en el $\operatorname{arco}(i, j)$. Se tiene

$$
\min \sum_{i \in V} \sum_{j \in V} c_{i j} x_{i j}
$$

Sujeto a:

$$
\begin{aligned}
& \sum_{i \in V} x_{i j}=1 \quad \forall j \in V \backslash\{0\} \\
& \sum_{j \in V} x_{j i}=1 \quad \forall j \in V \backslash\{0\} \\
& \sum_{i \in V} x_{i o}=K \\
& \sum_{j \in V} x_{o j}=K \\
& \sum_{i \notin S} \sum_{j \in s} x_{i j} \geq r(S) \quad \forall S \subseteq V \backslash\{0\}, S \neq 0 \\
& x_{i j} \in\{0,1\} \quad \forall i, j \in V
\end{aligned}
$$

La ecuación (4) considera la minimización de los costos de desplazamiento entre los nodos $(i, j)$ activados en la solución óptima encontrada.

Las restricciones (5) y (6) imponen que exactamente un arco ingresa y abandona cada vértice asociado con un consumidor respectivamente. Análogamente las restricciones (7) y (8) imponen el grado de requerimiento del vértice depósito. Estas restricciones reemplazan las de $2 \mid \mathrm{VI}-1$.

Las restricciones de capacidad-corte, CCCs (capacity-cut constraints) que se expresan en (9) imponen la conectividad de la solución con los requerimientos de capacidad del vehículo, ellas estipulan que cada corte definido por un conjunto de consumidores $S$ es cruzado por un número de arcos no menor que $r(S)$ (mínimo número de vehículos que se necesitan para atender el conjunto $S$ ). Siguen siendo válidas también si $r(S)$ es reemplazada por el límite inferior BPP.

La familia de restricciones (9) tiene cardinalidad exponencial que crece con el tamaño de $n$. Aquí se identifica la complejidad matemática de los modelos de ruteo, debido a que el número de restricciones de este tipo aumenta con base en $n$. La ecuación (10) expresa la naturaleza binaria de las variables de decisión del modelo. 


\section{CÁLCULO DEL CONSUMO DE COMBUSTIBLE}

A diferencia de otras emisiones de los vehículos, el $\mathrm{CO}_{2}$ es directamente proporcional al consumo de combustible $[4,5,6$, 7]. Las emisiones de los motores diésel varían según el tipo y la densidad de diésel que se utiliza. Diesel estándar emite $2.82 \mathrm{Kg} \mathrm{C} 02 /$ litro [8] y diésel ultra bajo en azufre $2.57 \mathrm{Kg}$ C02/litro [7]. En general, los factores de conversión del reporte ambiental de DETR (Department Of Enviromental Transport And The Regions) [9] afirma que las emisiones del diésel incluso las del diesel ultra bajo en azufre corresponden a $2.68 \mathrm{~kg} \mathrm{C02/litro.} \mathrm{Este} \mathrm{valor} \mathrm{es} \mathrm{muy} \mathrm{comparable} \mathrm{con} \mathrm{el} \mathrm{que}$ fijó la oficina australiana de efecto invernadero, en el que se afirma que aproximadamente $2.7 \mathrm{~kg} \mathrm{CO} \mathrm{C}_{2}$ son emitidos por litro de combustible diésel.

En la literatura que se enfoca en el estudio del consumo de combustible asociado a los problemas de ruteo se encuentran diferentes propuestas tales como: Apaydin y Gönüllü usan valores fijos para calcular el consumo de combustible [10]. Kuo calcula el consumo de combustible con respecto a la carga y velocidad del vehículo [11]. Jabali et al lo modelan como una función de la velocidad [12]. Bektaş y Laporte calculan el consumo de combustible basados en el promedio de velocidad y carga del vehículo [13]. Suzuki considera la carga del vehículo y los tiempos de espera en las ubicaciones de los clientes [14]. Ubaeda considera la carga del vehículo [15]. Xiao et al formula una ecuación lineal dependiente sobre la carga del vehículo [2]. Huang et al modelan una regresión lineal para el consumo de combustible basada en función de la carga y la distancia [16]. Demir et al $[17,18]$ y Liu y Helfand usan una ecuación matemática [19].

\section{A. CVRP incluyendo impacto ambiental.}

El G-VRP es el problema de ruteo de vehículos donde se considera el consumo de energía. Los costos de combustible tienen una parte significativa del transporte a base de petróleo [2]. Reducir el consumo de combustible y mejorar la eficiencia de la operación del transporte en el nivel operativo es un objetivo de gran importancia en la cadena de suministro. $\mathrm{Si}$ se reduce el consumo de combustibles de la industria del petróleo esto tiene efecto directo sobre la reducción de los gases de efecto invernadero [2,9]. Por lo tanto incluir el consumo de combustible en los modelos de ruteamiento verde se constituye en un aspecto de gran importancia a tener en cuenta para representar la situación real en la solución de los mismos [10]. De acuerdo con el informe del Departamento de Energía de Estados Unidos (2008), la velocidad de desplazamiento, el peso de la carga así como la distancia de transporte, son los factores que afectan el consumo de combustible. Los modelos matemáticos considerados en este trabajo son los propuestos en $[2,20]$.

Jaramillo [20] propone un modelo matemático lineal entero mixto para el CVRP que denomina GVRP (Green Vehicle Routing Problem). El planteamiento matemático que propone considera la siguiente notación: $d_{i j}$ corresponde a la distancia entre los nodos $i$ y $j ; x_{i j}$ es una variable binaria que toma el valor de 1 si se utiliza el arco que une los nodos $i$ y $j$ y toma el valor de cero en caso contrario; $C W$ corresponde al peso del vehículo sin carga; $Q$ representa la capacidad de peso del vehículo; $y_{i j}$ indica el peso total del vehículo considerando la carga que se está transportando; $q_{j}$ corresponde a la demanda del cliente $j$ representada en peso; $L$ representa el número total de nodos.

$$
\begin{aligned}
& \min \sum_{i=1}^{L} \sum_{j=1}^{L} d_{i j} y_{i j} \\
& \text { s.a } \\
& y_{i 1}-C W x_{i 1} \geq 0 \quad \forall i \\
& (C W+Q) x_{i j}-y_{i j} \geq 0 \\
& \sum_{i=1}^{L} y_{i j}-\sum_{i=1}^{L} y_{j i}=q_{j} \quad J=2, \ldots, L \\
& y_{i j} \geq 0 \quad \forall i, j \\
& x_{i j} \in[0,1]
\end{aligned}
$$

La función objetivo (11) consiste en minimizar la distanciapeso cuantificada en todas las rutas que constituyen las solución óptima. El conjunto de restricciones (12) garantiza que el vehículo llegue al depósito vacío, es decir que el peso del vehículo sea igual a $\mathrm{CW}$. El conjunto de restricciones (13) corresponde a las conexiones de las variables $x_{i j}$.El conjunto de restricciones (14) corresponde a la conservación del flujo. Las restricciones (15) y (16) se refieren a la naturaleza de las variables.

\section{UN EJEMPLO DEL PROBLEMA CVRP Y GVRP.}

En esta sección se muestra un ejemplo numérico para mostrar la solución del problema con y sin impacto ambiental.

Se consideran 10 clientes y un depósito desde donde son atendidos, en la tabla 1 se presenta la información.

Tabla 1. Datos ejemplo 


\begin{tabular}{|c|c|c|c|}
\hline \multirow{2}{*}{ Nodo } & \multicolumn{2}{|c|}{ coordenadas } & \multirow{2}{*}{ q } \\
\cline { 2 - 3 } & $\mathbf{x}$ & $\mathbf{y}$ & \\
\hline 1 & 46 & 54 & 0 \\
\hline 2 & 85 & 54 & 4 \\
\hline 3 & 90 & 4 & 1 \\
\hline 4 & 56 & 87 & 1 \\
\hline 5 & 37 & 15 & 3 \\
\hline 6 & 24 & 65 & 2 \\
\hline 7 & 15 & 6 & 3 \\
\hline 8 & 53 & 24 & 4 \\
\hline 9 & 26 & 38 & 2 \\
\hline 10 & 86 & 77 & 2 \\
\hline 11 & 8 & 80 & 1 \\
\hline
\end{tabular}

El nodo 1 corresponde al depósito donde se considera su demanda igual a cero, los nodos 2 al 11 corresponden a los 10 clientes que deben ser servidos. En la próxima sección se presentan los detalles de los modelos matemáticos que son utilizados.

A. Modelos matemático considerado para resolver el CVRP y el GVRP

Con base en el modelo matemático presentado por Jaramillo para el CVRP [20] definido por las ecuaciones (11) a (16), que a su vez se basa en el modelo VFF descrito en la sección 2 se presenta un modelo CVRP que combina los dos modelos anteriores con las restricciones denominadas MTZ para evitar la formación de ciclos dentro del tour [21].

Se consideran los siguientes parámetros: $d_{i j}$ corresponde a la distancia entre los nodos $i$ y $j ; q_{i}$ demanda del cliente $i$ en unidades de peso; $Q$ Peso del vehículo. Se define $x_{i j}$ como una variable binaria que toma el valor de 1 si el vehículo visita el cliente $j$ después de visitar el cliente $i$ y cero en caso contrario. $L, U$ representan el número total de clientes y subconjuntos de clientes respectivamente y $\mu_{i}$ es un valor arbitrario.

$$
\begin{aligned}
& \min \sum_{i=1}^{L} \sum_{i=1}^{L} d_{i j} x_{i j} \\
& \sum_{j=2}^{L} x_{1 j}=k \\
& \sum_{i=2}^{L} x_{i 1}=k \\
& \sum_{i=1}^{L} x_{i j}=1 \quad j=2, \ldots, L ; i \neq j \\
& \sum_{j=1}^{L} x_{i j}=1 \quad j=2, \ldots, L ; j \neq i \\
& \sum_{(i, j) \in A: i \in U, j \in(L-U)} x_{i j} \geq 1 \quad 2 \leq|U| \leq|L|-2
\end{aligned}
$$

$q_{i} \leq \mu_{i} \leq Q$

$x_{i j} \in[0,1]$

$\mu_{i} \geq 0$

La función objetivo (17) minimiza el total de la distancia recorrida por todos los vehículos. Las restricciones de tipo (18) y (19) garantizan respectivamente que las $k$ rutas inicien y terminen en el nodo 1 que se considera como el depósito.

Las restricciones (20) indica el ingreso de la ruta al cliente $i$ y (21) Garantizan la salida de la ruta desde el cliente $i$. La restricción (22) corresponde a las restricciones de eliminación de sub-tours de Miller-Tucker-Zemlin que junto con las restricciones del tipo (23) garantizan que las rutas no excedan la capacidad de los vehículos. Finalmente las restricciones (24) y (25) definen la naturaleza de las variables.

La figura 1 corresponde a la solución del CVRP considerando el modelo descrito por las ecuaciones (17) a (25). La figura 2 corresponde al GCVRP considerando las ecuaciones (11) a (16). Como se puede observar cuando se considera el impacto ambiental la distancia recorrida aumenta en $21.29 \mathrm{~km}$, pero las emisiones ambientales se disminuyen en $272.02 \mathrm{~km}$-ton.

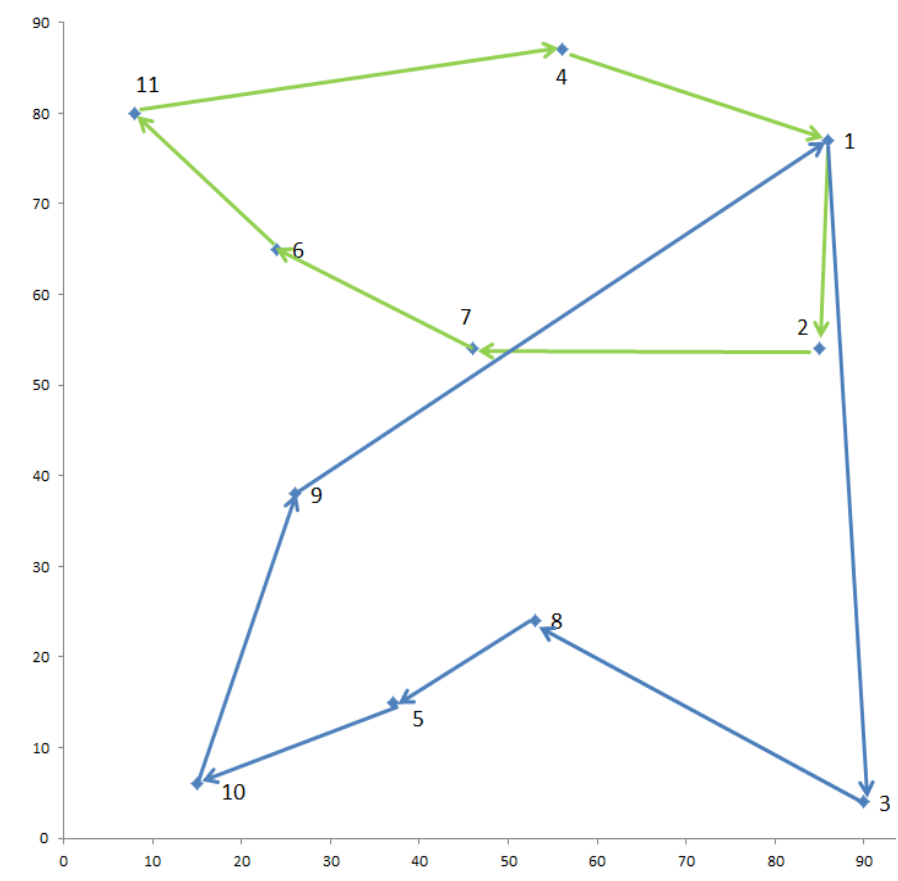

Figura 1. Solución del CVRP.

Lectura de resultados de la Figura 1.

$\mathrm{R} 1=\left[\begin{array}{lllll}3 & 8 & 5 & 10 & 9\end{array}\right], \mathrm{R} 2=\left[\begin{array}{lllll}2 & 7 & 6 & 1 & 4\end{array}\right]$

Distancia $=451.37 \mathrm{~km}$ Distancia-peso $=5959.2 \mathrm{~km}$-ton 
Lectura de resultados de la Figura 2.

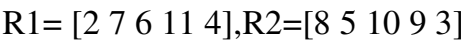

Distancia $=472.66 \mathrm{~km}$ Distancia-peso $=5687.18 \mathrm{~km}$-ton

Este ejemplo muestra que a pesar de tratarse de un sistema de transporte pequeño (11 nodos), se trata de un problema NPDuro. Generalmente cuanto este tipo de problemas requieren ser resueltos, las técnicas exactas resultan ser poco recomendables debido a que se pueden presentar dos situaciones, la primera que se requiera de un tiempo computacional excesivo para entregar la solución óptima o la segunda que la metodología no converja. Esta es la razón que motiva la propuesta de este trabajo que consiste en presentar una metodología híbrida que combina una técnica exacta con una metaheurística.

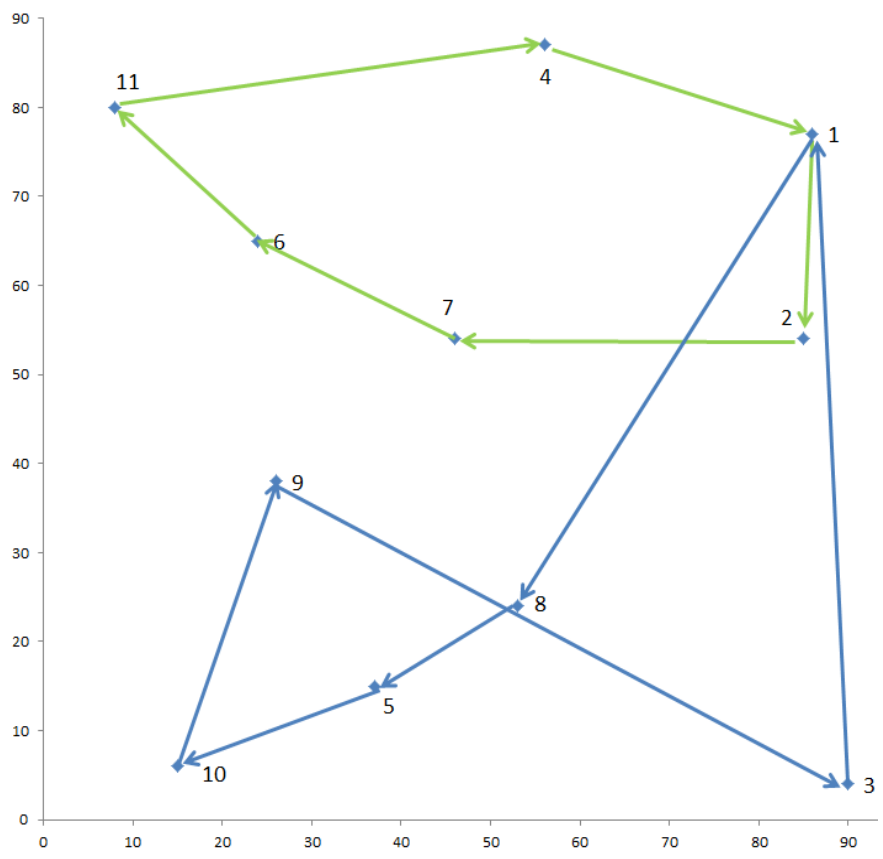

Figura 2. Solución del GVRP

\section{METODOLOGÍA DE SOLUCIÓN PROPUESTA}

Se presenta inicialmente la codificación utilizada, donde se considera la secuencia de visita de los clientes y la demanda en peso de cada uno de ellos. Luego se describe la metodología propuesta basada en la estrategia de rutear primero y asignar después, donde el proceso de ruteo se resuelve de forma exacta encontrando la solución óptima del TSP asociado a la instancia propuesta. La etapa de asignar después se resuelve mediante un algoritmo memético.

\section{A. Etapa 1: Rutear primero}

En esta etapa se resuelve el problema como un TSP incluyendo todos los nodos, mediante el modelo matemático denominado MTZ [22] utilizando el CPLEX.

\section{B. Etapa 2: Asignar Después}

En esta etapa que es resuelta utilizando un algoritmo memético, se construye el conjunto de alternativas de solución con base en la secuencia óptima encontrada en la etapa anterior y a partir de ello se desarrollan todos los pasos del algoritmo.

1. Codificación: Representación de una solución.

La secuencia óptima obtenida en la etapa anterior se representa mediante un vector donde cada elemento representa un nodo, considerando el nodo 1 como el nodo depósito. Si se tiene un vector como el que se presenta en la figura 3 , su lectura corresponde a una ruta que inicia en el depósito, el primer cliente que se visita al salir del depósito es el 6, y de forma secuencial se van visitando los clientes restantes hasta finalizar en el cliente 9. Cuando se visita el último cliente se retorna al depósito.

$$
\begin{array}{cccccccccccc}
1 & 6 & 11 & 4 & 10 & 2 & 3 & 8 & 5 & 7 & 9 & 1 \\
& \multicolumn{1}{c}{\text { Figura 3. Vector solución. }} & & &
\end{array}
$$

Con base en la secuencia obtenida se divide el vector en varias secciones, donde cada una de ellas representa una ruta, el criterio de finalización de la ruta corresponde a la capacidad $Q$ del vehículo utilizado,tal como se muestra en la figura 4.

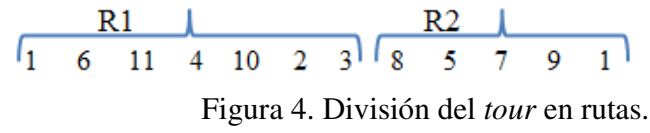

2. Construcción de la población inicial: Tomando como base la solución de la etapa anterior, la secuencia obtenida es recorrida de izquierda a derecha sumando en cada consumidor la carga que tiene que ser atendida siempre y cuando no exceda la capacidad del vehículo. Cuando se alcanza la capacidad del vehículo, se almacena la ruta y se inicia una nueva ruta que atienda la demanda de los consumidores restantes. El procedimiento se describe en la figura 5.

3. Selección: Se utiliza la selección por torneo. En esta estrategia se eligen las configuraciones realizando 2 torneos considerando toda la población [23]. 


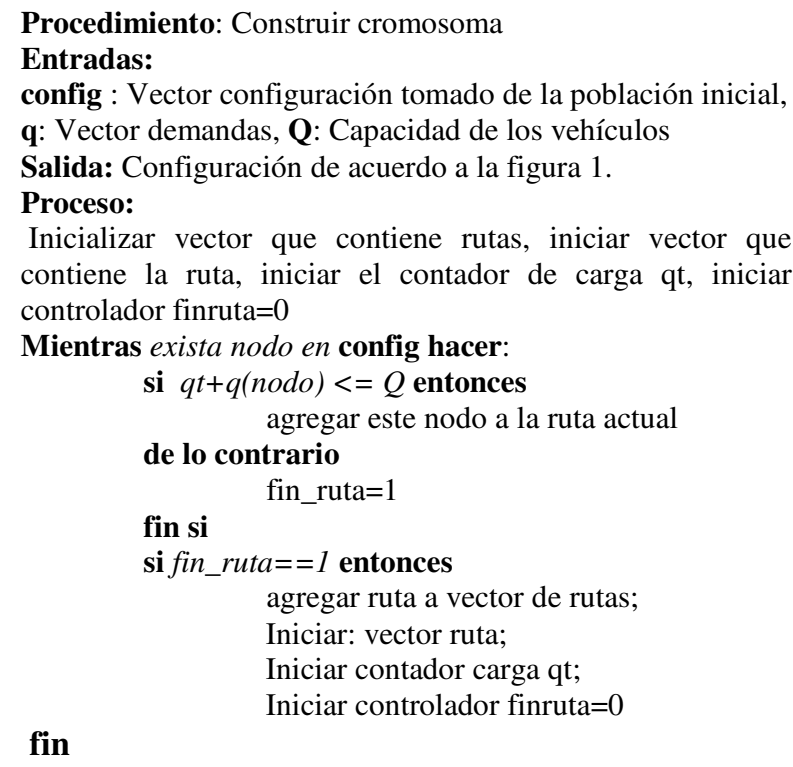

Figura 5. Procedimiento de creación de cromosomas En cada torneo son seleccionados un conjunto de $k$ configuraciones. Realizando $n$ juegos se termina el proceso de selección. En esta implementación se usan 2 torneos con $k=$ 2 , en cada torneo se elige una configuración padre. Obtenidas las dos configuraciones padre se procede a recombinarlas para generar dos nuevas alternativas de solución.

\section{Cruzamiento}

En la etapa de cruzamiento se utiliza la filosofía del cruzamiento EPX que consiste en pasar una parte de la información genética del padre 1 y mapear la información del padre 2 de forma que no se repitan características que ya se han considerado. En esta propuesta se considera un punto de cruce tal como se muestra en el siguiente ejemplo.

$$
\begin{array}{llllllllll}
\mathrm{A}= & 1 & 2 & 3 & 4 \mid & 5 & 6 & 7 & 8 & 9 \\
\mathrm{~B}= & 8 & 5 & 6 & 7 \mid & 2 & 9 & 1 & 3 & 4
\end{array}
$$

Paso 1: Generar de forma aleatoria un punto de cruce. En este caso corresponde a la posición 4.

Paso 2: Generación de las dos nuevas configuraciones

$$
\begin{array}{llllllllll}
\mathrm{A}^{\prime}= & 1 & 2 & 3 & 4 \mid & 2 & 9 & 1 & 3 & 4 \\
\mathrm{~B}^{\prime}= & 8 & 5 & 6 & 7 \mid & 5 & 6 & 7 & 8 & 9
\end{array}
$$

Paso 3: Debido a que se puede producir repetición de la información genética se hace una reparación del cromosoma de acuerdo a la correspondencia entre elementos observadas en la configuración inicial. La posición 1 en el cromosoma A corresponde al valor 1 y la misma posición en el cromosoma $\mathrm{B}$ es corresponde al valor 8 , por tanto 1 corresponde a 8 . Con base en esa regla se reestructuran los cromosomas y se generan las dos nuevas configuraciones.

$$
\begin{array}{llllllllll}
\mathrm{A}^{\prime \prime}= & 1 & 2 & 3 & 41 & \mathbf{5} & 9 & \mathbf{8} & \mathbf{6} & \mathbf{7} \\
\mathrm{B}= & 8 & 5 & 6 & 71 & \mathbf{2} & \mathbf{3} & \mathbf{4} & \mathbf{1} & \mathbf{6}
\end{array}
$$

5. Mutación: Se aplica una mutación especializada explicada en la referencia [24], sobre toda la población. Se denomina Dmutation. Se explica mediante un ejemplo.

Se tiene el cromosoma

$$
\begin{array}{llllllllll}
A= & 1 & 3 & 5 & 7 & 4 & 6 & 8 & 9 & 2
\end{array}
$$

Paso 1: Se genera una posición a mutar de forma aleatoria, aquí la posición a mutar es la 4 que corresponde al cliente 7;

Paso2: Se genera un gen (nombre de un cliente) de forma aleatoria, aquí el cliente 9.

Paso 3: Se ubica el cliente, en este caso el cliente 9 en la posición del cliente 7 .

Paso 4. El cliente que corresponde a la posición 4, es el cliente 7 el cual es ubicado en la posición del cliente 9. El cromosoma mutado queda con la siguiente codificación.

$$
\mathrm{A}^{\prime}=\begin{array}{lllllllll}
1 & 3 & 5 & 9 & 4 & 6 & 8 & 7 & 2
\end{array}
$$

En la figura 6 se presenta el diagrama global de flujo de la metodología propuesta.

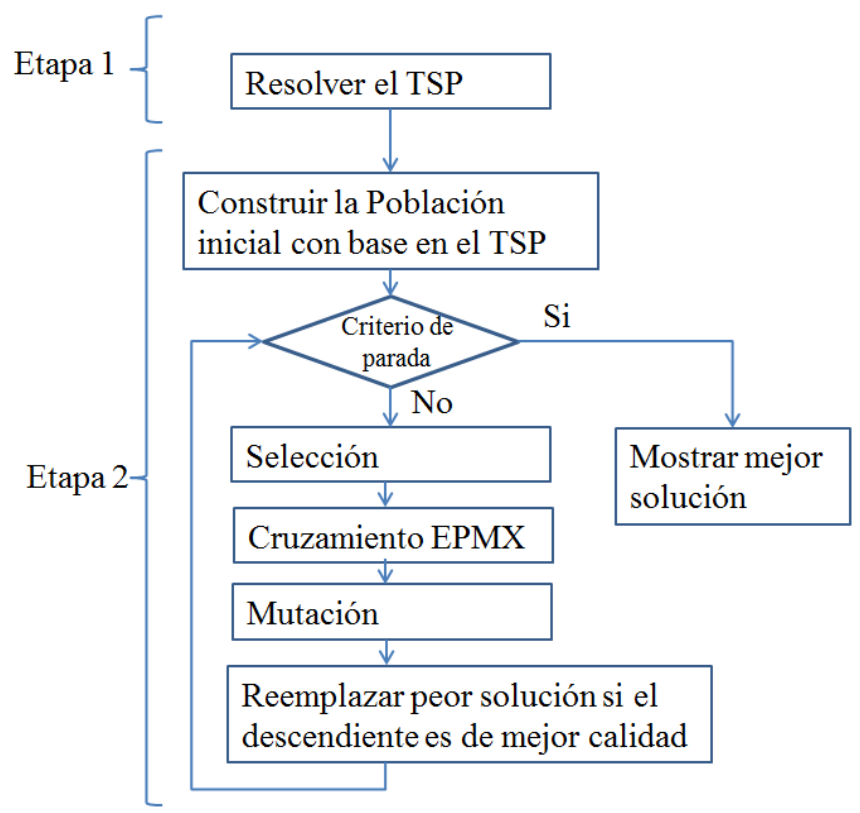


Figura 6. Metodología de solución considerando impacto ambiental.

\section{RESULTADOS}

Para validar la metodología propuesta se consideran casos de prueba que pueden ser descargados en [24]. En la definición de la instancia $\mathrm{n}$ corresponde al número de clientes, $\mathrm{k}$ corresponde a la cantidad de vehículos disponibles y Q corresponde a la capacidad del vehículo. En la tabla 2 se presentan los resultados obtenidos.

Tabla 2. Resultados Obtenidos

\begin{tabular}{|c|c|r|r|r|r|}
\hline Instancia & Q & \multicolumn{2}{|c|}{ CVRP } & \multicolumn{2}{c|}{ GVRP } \\
\cline { 3 - 6 } & & $\begin{array}{c}\text { Dist } \\
(\mathrm{Km})\end{array}$ & $\begin{array}{r}\text { Dist-peso } \\
(\text { Km-ton })\end{array}$ & Dist (Km) & $\begin{array}{r}\text { Dist-peso } \\
\text { (Km-ton) }\end{array}$ \\
\hline P-n16-k8 & 35 & 451.3 & 6813.9 & 451.9 & 6101.3 \\
\hline P-n19-k2 & 160 & 212.6 & 17398.1 & 270.78 & 13219.9 \\
\hline P-n20-k2 & 160 & 217.41 & 17667.61 & 252,85 & 13668.24 \\
\hline
\end{tabular}

Los resultados obtenidos para el CVRP en las instancias probadas alcanzan los resultados publicados en [24] lo que indica que la metodología propuesta es confiable y eficiente en este tipo de problema.

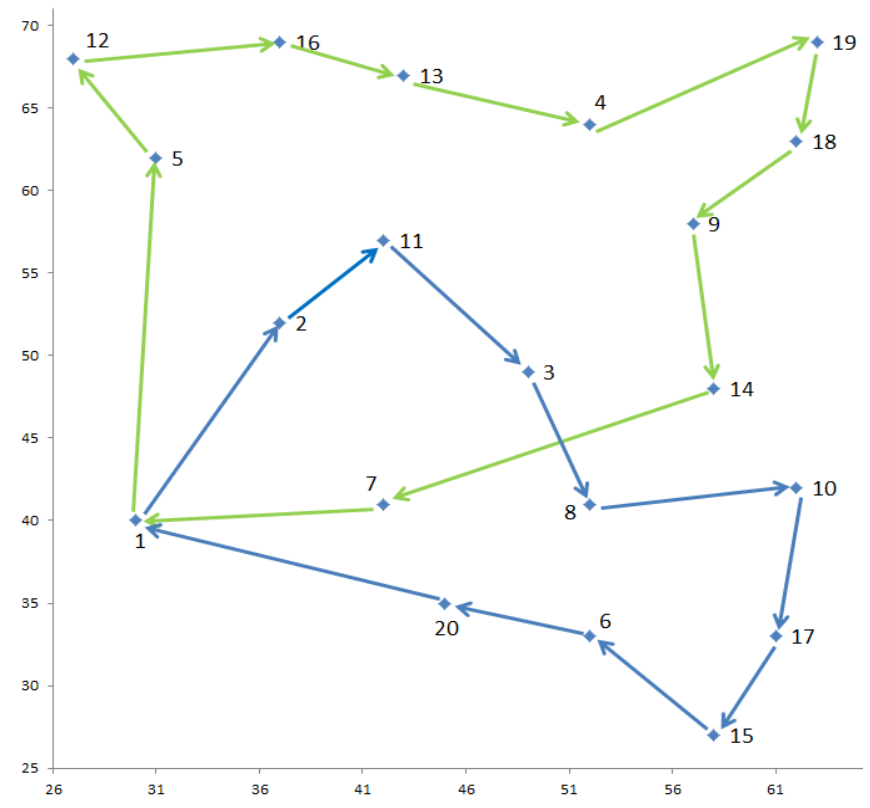

Figura 7. Caso P-n20-k2 . CVRP

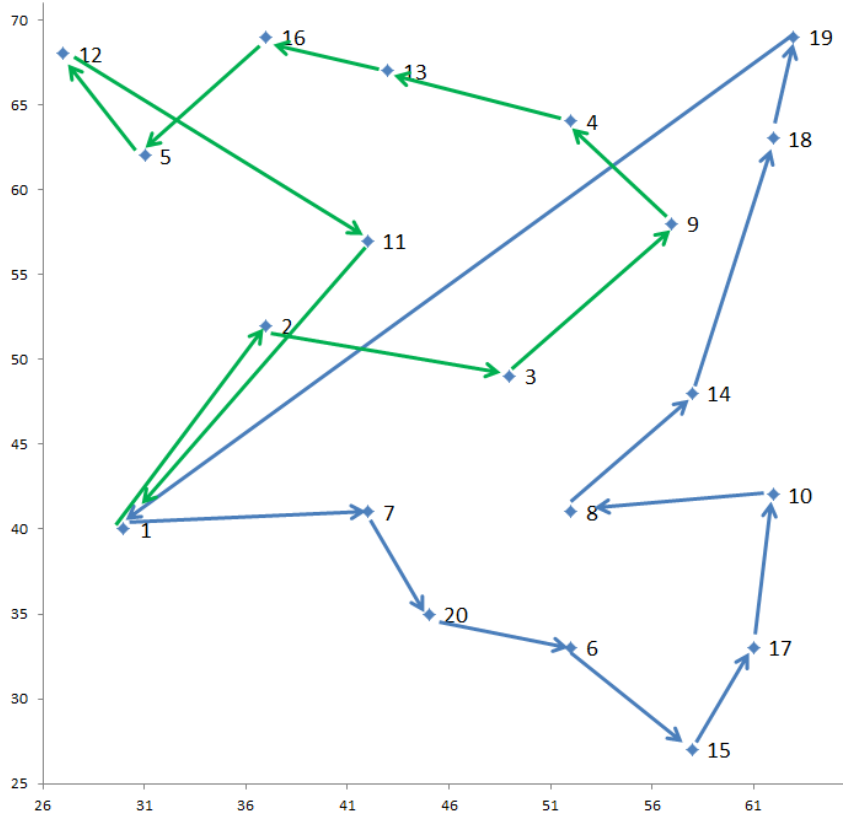

Figura 8. Caso P-n20-k2 GVRP.

La metodología propuesta fue codificada de la siguiente forma: Para la etapa 1 se implementó un script usando Matlab, Ampl y CPLEX, para la etapa 2, se implementó el Algoritmo Genético en Matlab. Los resultados se obtuvieron utilizando un equipo con un procesador Intel(R) Core(TM) i5 cuatro núcleos y un sistema operativo Windows 7 Professional 64bits.

En la figuras 7 y 8 se presentan los resultados obtenidos para el CVRP y el GVRP respectivamente de la instancia P-n20$\mathrm{k} 2$.

En la figura 7 se representan los resultados obtenidos al resolver el CVRP considerando el modelo descrito por las ecuaciones (17) a (25), que se leen como:

Ruta 1: [5 12161341918914 7]

Ruta 2: [2 11381017156 20]

Distancia recorrida: $217.41 \mathrm{~km}$

Distancia-peso: $17667.61 \mathrm{~km}$-ton

Con base en la evaluación del modelo GCVRP considerando las ecuaciones (11) a (16) se obtienen los resultados que se presentan en la figura 8 , que se leen como:

Ruta 1: [7 20615171081418 19]

Ruta 2: [2 394131651211 ]

Distancia recorrida: $252.85 \mathrm{~km}$

Distancia-peso: $13668.24 \mathrm{~km}$-ton 
A. Análisis de resultados

No es posible obtener respuestas a través de los modelos matemáticos exactos cuando se consideran instancias de más de 15 clientes. La metodología propuesta es una alternativa de solución para instancias con estas características.

La metodología propuesta mostró ser eficiente para casos de mayor tamaño, donde se encuentra la solución para ambos problemas en tiempos computacionales aceptables. En las respuestas encontradas se observa que los valores de la función objetivo que se encuentra para el CVRP dista de la encontrada cuando se considera el GCVRP cuando son evaluadas bajo las dos funciones objetivo bajo estudio.

\section{CONCLUSIONES Y TRABAJOS FUTUROS}

Se plantea una metodología híbrida que resuelve el CVRP en dos etapas, inicialmente se resuelve de forma exacta el problema de TSP. A partir de la secuencia obtenida se construye la población inicial que dará origen a un algoritmo genético modificado para encontrar la solución del problema GCVRP.

Es recomendable evaluar otras estrategias en la etapa de cruzamiento, así como estrategias de mejoramiento y búsqueda local aplicadas en la etapa de mutación o como complemento a la etapa de cruzamiento.

Debido a que se están considerando dos objetivos en conflicto podría evaluarse la opción de explorar estrategias multiobjetivo en la solución del problema.

\section{REFERENCIAS}

[1]. I. Kara, B.Y. Kara and M.K. Yetis, "Energy minimizing Vehicle Routing Problem". In Dress, A., $\mathrm{Xu}$, Y.,Zhu, B. (Eds). Combinatorial Optimization and Applications, Lecture Notes in Computer Sciencie, vol.4616. Springer, Berlin/Heidelberg, 2007. pp 62-71.

[2]. Y. Xiao, Q. Zhao, I. Kaku, Y. Xu. " Development of a fuel consumption optimization model for the capacitated vehicle routing problem".Computers\& Operations Research, 39:1419-1431,2012
[3]. P.Toth, D.Vigo. "The Vehicle Routing Problem".SIAM. Bologna,Italy.2002

[4]. J.H. Kent, N.R. Mudford. "Motor vehicle emissions and fuel consumption modelling". Transportation Research Part A: General 13(6):pp395-406. Dec 1979.

[5]. M.Schingh,E. Brunet, and P.Gosselin. "Canadian new light duty vehicles: trends in fuel consumption and characteristics (1988-1998)". Office of Energy Efficiency, Natural Resources, Canada. Jul 2000.

[6]. P.Romilly. "Substitution of bus for car travel in urban Britain: an economic evaluation of bus and car exhaust emission and other costs". Transportation Research part D:Transport and Enviroment 4(2):1999. pp 109-125.

[7]. B. Hutton. "The importance of relating fuel consumption rates and driving cycles. Measuring, modelling, monitoring and motivation changes in fuel consumption". Transport Research Institute worshop.Edinburgh.Sep 2002.

[8]. J. Sainsbury plc. "Being greener: environment report 2002". London 2002.

[9]. M. A. Figliozzi. "An iterative route construction and improvement algorithm for the vehicle routing problem with soft time windows". Transportation Research Part C: Emerging Technologies, 18(5),668679. 2010. Doi:10.1016/j.trc.2009.08.005

[10]. ÖApaydin, MT Gönüllü. "Emission Control with route optimization in solid waste collection process: A case study".Sadhana, 33(2):71-82,2008.

[11]. Y. Kuo."Using Simulated Annealing to minimize fuel consumption for the time-dependent vehicle routing problem". Computers and Industrial Engineering, 59:157-165,2010

[12]. O. Jabali, T Van Woensel, A.G. Kok. "Analysis of travel times and $\mathrm{CO}_{2}$ emissions in the time-dependent vehicle routing". Production and Operations Managment, 21(6):1060-1074.2012

[13]. T.Bektaș, G. Laporte."The pollution routing problem". Transportation Research part B,45:12321250,2011

[14]. Y. Suzuki." A new trucking approach for reducing fuel consumption and pollulantemission": TransportationResearchPart D

[15]. S. Ubeda, F.J. Arcelus, J. Faulin."Green Logistics at Eroski: A case study". International Journal of Production Economics, 131:44-51,2011.

[16]. Y. Huang;C. Shi, L. Zhao, T.Van Woensel. "A study on carbon reduction in the vehicle routing problem with simultaneous pickups and deliveries. IEEE 
International Conference on Service Operations and Logistics and Informatics. 8-10 july 2012. Suzhou,China.

[17]. E. Demir,T.Bektaș,G. Laporte. "A comparative analysis of several vehicle emission models for road freight transport". TransportationResearchPart D, 16:347-357,2011.

[18]. E. Demir,T.Bektaș,G. Laporte. "Anadaptativelargeneighbor Hood searchheuristicforthepollutionroutingproblem". EuropeanJournal of OperationResearch,223:346$359,2012$.

[19]. Y. Liu,G.E. Helfand. "The alternative motor fuel sact, alternative-fuel vehicles, and greenhouse gas emissions". TransportationResearchPart A,43:755$764,2009$.

[20]. J.R Jaramillo. The Green Vehicle Routing Problem. Southeastern INFORMS Proceedings. pp 470-477. Oct 2011.

[21]. C. E. Miller, A. W. Tucker, and R. A. Zemlin. "Integer programming formulations and traveling salesman problems," J. ACM, 7 (1960), pp. 326-329.

[22]. R. Gallego, A. Escobar, E. Toro. "Técnicas metahuerísticas de Optimización". Universidad Técnológica de Pereira. Segundaedición. Pp 136.

[23]. Z. TAO. “ TSP Problem solution based on improved Genetic Algorithm TSP Problem solution based on improved Genetic Algorithm”. Fourth International Conference on Natural Computation. IEEE 2008.

[24]. P. Augerat, J.M. Belenguer, A. Corberan; A. Naddef; D.RinaldiG. "Computational Results with a Branch and Cut Code for the Capacitated Vehicle Routing Problem". Research Report 949-M, Université Joseph Fourier, Grenoble, France. 1998 